\title{
1. Digital Literacies and the National Broadband Network: Competency, Legibility, Context
}

\section{Introduction}

In 2010 we conducted a pilot study ${ }^{1}$ in North Hobart and Midway Point Tasmania, one of the first Australian suburbs to be offered trial services from the National Broadband Network $(\mathrm{NBN})$. The study was conducted in the very earliest stage of the rollout, at a point where only 'innovators' and perhaps the first of the 'early adopters' - to use technology diffusion terms (Rogers, 1983) - had actually experienced the service. These early adopters were, nevertheless, more accidental than the purposive technology enthusiasts characterising the adoption model, by way of chance in living in a trial location. The objective of the pilot study was to get a clearer view of these householders' presumptions of the NBN and their expectations of high-speed broadband (HSB), and, concomitantly, to get a clearer view of their motivations for adoption or non-adoption (Wilken at al., 2011). The pilot also served to inform an ongoing longitudinal study of the implications of broadband consumption on household media ecologies in Brunswick, Victoria, ${ }^{2}$ a second NBN pioneer suburb that is one of five first release test sites on mainland Australia.

The study of household adoption, expectation and use of HSB was chosen because the home will be a crucial site for the success of the NBN and growth of the digital economy (Apperley et al., 2011). The NBN is premised on a fibre-to-the-home (FTTH) installation model. Apart from the financial viability of the network depending upon high rates of household take-up, the broader ambition of the network for inclusion, connectivity and productivity (Conroy, 2009) depends upon how this technology infrastructure and access is understood and engaged with by householders. That is, upon the achievement of digital literacy capacities required to live in NBN-connected homes and to make the most of NBNconnected living.

Key themes emerging from this research are presented in this paper through the metaphor of 'digital literacy'.

\section{How is digital literacy defined?}


As a metaphor, digital literacy has its problems. What, after all, is the digital literature? What is the digital canon and what is the skill-set required to comprehend that canon, where, unlike literacy in text, skills are highly specific and highly mutable? Is it sufficient to be fluent in the use of the Windows operating system, or do the truly erudite use Linux line commands? Am I literate if I can Photoshop an image or edit a video file, but don't know my way around Illustrator or Garageband? Suppose I can use all those applications, but I can't use HTML, let alone read and write $\mathrm{C}$ ? In conditions where there can be no agreement on the corpus, and no master skill-set to comprehend that corpus, it is not surprising that definitions of digital literacy differ. Despite these difficulties though, 'digital literacy' references 'literacy' with sufficient clarity to make it useful, and we use it here because like text literacy, digital literacy implies a requisite compound of instrumental skills, semantic skills, and social skills.

The Australian Communications and Media Authority (ACMA) define digital literacy, based on the UK's OfCom (2009) definition, as 'the technical and intellectual skills to access, understand, and participate in or create content on digital media and communications technologies' (ACMA, 2008: 51). This reflects a more instrumental definition that emphasises technical competency as an individually acquirable attribute. This form of literacy is technology centred, and relates to competencies that include using various software applications, to information searching, navigating, assessing, and communicating (Hobbs, 2008; Luke, 2000, 2001).

The European Commission on the other hand, emphasises semantic rather than instrumental skills, and directs literacy at command of media content rather than delivery technologies. They define media literacy as,

the ability to access, analyse and evaluate the power of images, sounds and messages which we are now being confronted with on a daily basis and are an important part of our contemporary culture, as well as to communicate competently in media available on a personal basis. Media literacy relates to all media, including television and film, radio and recorded music, print media, the Internet and other new digital communication technologies. (European Commission, 2009)

This form of literacy emphasises a skill-set that bears upon command of the message rather than its media, and relates to competencies associated with making meaning and interpreting meaning. It is a definition inherited from print and mass media milieus of media production, 
where media literacy is understood as the capacity to critically read, rather than make, and which is then transposed onto newer digital media texts.

Thirdly, the term 'soft skills' (Jenkins, 2006, 2009; Livingstone, 2009) is used to describe the socially oriented competencies necessary to read, participate in or contribute to online content and digital life. This understanding of digital literacy decentres the individual and her command of technology, or lack thereof, in order to focus on the communicative, interactive, networked, or social nature of her media production and consumption. Digital literacy is understood as a social skill-set that addresses the shift from mass media - characterised by centralised organisation and clear distinctions between production and consumption - to new media, and its possibilities for accessible, participatory and dispersed forms of content production, distribution and consumption.

The literacy metaphor, and its varying definitions, suggest that a range of instrumental, semantic and social knowledges, skills, and capabilities need to be developed to participate in an increasingly digitally mediated world, in which digital resources have become more integrated into economic and social life. The metaphor suggests that just as an inability to use the written word has long posed a significant barrier to a full participation in print-based cultures, so an inability to use digital resources poses a similar barrier in contemporary digital cultures, and in this regard, the digital literacy metaphor earns its place.

The significance of digital literacy to contemporary life is captured and deployed in discussions of the digital economy. The digital economy is used to describe changing systems of production and consumption resulting from the availability and use of broadband infrastructures, digital networks and new technology. Yet, the digital economy is not simply a commercial construct, but encompasses the raft of business transactions, government services, and social exchanges that emerge from the take-up and usage of digital media (e.g. ACMA, 2007, 2009).

In a digital economy, many everyday and essential economic activities - along with the delivery of important social services including health, education and e-government - occur online and through multiple media devices. In this context digital literacy is important for the achievement of economic productivity and prosperity, but also for fostering greater cultural participation, social inclusion and personal connection (Foth et al., 2011). 
In Australia, increased levels of basic access to the Internet, rapidly increasing penetration of digital technologies in households, and widespread migration of commerce and services online have shifted the terms of the debate about digital literacy from instrumental literacy alone, to a more nuanced position that takes account of semantic and social factors. The focus is no longer on 'digital divides', read in terms of an 'access vs no-access' binary, to a consideration of 'participation gaps' and the varied qualities of use based on abilities to genuinely participate in, understand and contribute to digitally mediated social, economic and cultural life (e.g. Livingstone, 2009; Maher, 2008; Warschauer, 2003).

Andrew Maher, of Infoxchange Australia, notes, "civic and democratic societies demand that everyone be able to access and share information. In the twentieth century this meant being able to read and having access to print materials, radio and television. In the twenty-first century it means having access to information and communications technology; in short, it means digital inclusion" (Maher, 2008). Gurstein goes further, taking up this theme in a number of publications to point out that the issue is 'effective use', not simply 'access', arguing that 'the concepts and strategies underlying the notion of the Digital Divide [are] little more than a marketing campaign for Internet service providers' (Gurstein, 2003, 2004).

Digital literacy implies effective use, and, in this context, is now viewed by researchers and policy makers as a critical aspect of social inclusion (e.g. ACMA 2007, 2009). By extension, for the success of the NBN digital literacy is viewed as critical in fostering effective participation in the digital economy, effective deployment of social services, effective participation in civil society, and effective participation in contemporary forms of sociality (e.g. DBCDE 2011).

A focus on digital literacy and effective participation also implies that the NBN's $\$ 45$ billion project to provide fibre-optic cable infrastructure to the front door of every home is not enough. How high speed broadband (HSB) is imagined, adopted, appropriated, domesticated and folded into routine by families and households who vary widely in their 'digital literacy' will be critical to its success, and critical to people's experience of digital life.

Despite this diversity, we return to a more critical view of digital literacy and question the assumptions that underpin the argument that digital literacy is the precursor to effective use of HSB through the NBN. Effective use of HSB, said to rely upon and to be fostered through increased digital literacy, is a 'deficit model' of causality, attributing non-use, marginal use, or ineffective use, to a personal deficit in technical, semantic or social forms of digital 
literacy, when it may well be that non-participation is not attributable to digital illiteracy, but arises instead from a rational decision to exclude, avoid, ignore or even subvert digital participation (e.g. Satchell and Dourish, 2009; Selwyn, 2003; Verbeek, 2005). As Satchell and Dourish (2009) note, "non-use is not an absence or a gap; it is not negative space. Nonuse is, often, active, meaningful, motivated, considered, structured, specific, nuanced, directed, and productive" (15).

The deficit model of digital literacy also assumes that effective use is contingent upon variable properties of the user - upon the 'literacy' of the user - when it would appear evident that variable properties of the 'literature', in this case, of the NBN and its HSB services, also have a bearing on effective use. Indeed, some technologies are illegible, even to literate users.

A third criticism of the use of the deficit model of digital literacy pulls back from agency, from a perspective that frames a personal interaction with technology, so that structural, socio-economic and cultural factures are also included in the frame of reference. Villagers without electricity do not eschew the Internet because they are digitally illiterate. In the case of Australia's NBN, electricity supply is not a problem, but, as we shall see, other structural factors relating to installation policies, for example, need to be considered.

\section{Method}

This paper reports on findings from an ethnographic study of 15 participant households in North Hobart and Midway Point, Tasmania. The North Hobart households (of mixed composition), ${ }^{3}$ had Fibre-to-the-Home (FTTH) provided in 2009 in advance of the NBN rollout, through the trial TasCOLT - Tasmanian Collaborative Optical Leading Testbed project (TECC, 2008; Spring and Wiatr, 2006; Given, 2010), ${ }^{4}$ whilst the Midway Point households had FTTH provided in 2010 as one of the first release test areas under the NBN rollout.

Our study involved the use of a number of qualitative methods spread over a one-year period. These involved established interview techniques as well as more novel methods, including 'technology tours' and the use of a 'domestic probe' pack (see Arnold 2004; Arnold, et al., 2006a; Arnold, et al., 2006b; Gaver, et al., 1999; Gaver, et al., 2004; Shepherd, et al., 2007). The first 'technology tour' occurred in early 2010 when participants were asked 
to escort us around the home, provide an inventory of the technologies in the home, and explain something of their origins, purposes, and use. Families were subsequently introduced to a 'Domestic Probe' pack containing a Polaroid camera and a 'Scrapbook', to record, assess, and reflect on their experiences with the HSB technologies. For a period of a year following this initial visit, a series of three follow-up face-to-face interviews was undertaken. Guided by the traces in the domestic pack, open-ended conversations were designed to elicit reflective insights into daily uses and expectations of HSB technologies and services in the home.

The study also draws on qualitative interview data from 20 households collected as part of the ongoing and larger study of the NBN roll-out in Brunswick. Participants were based on a purposive sample of various household internet connection types, drawn from a quantitative survey and analysis ${ }^{5}$. Interviews were semi-structured around household experience, motivations and practices of HSB appropriation and decision-making.

\section{Findings}

In our conversations with the people of North-Hobart and Midway Point, we found evidence of digital illiteracy, and also evidence of the weaknesses of digital literacy as an explanatory trope. We group these findings using three themes - (1) competency, or the presence of instrumental literacy, (2) legibility and illegibility in relation to the NBN and its HSB services, and (3) context, or the structural conditions limiting the acquisition of the NBN and its HSB services. These three themes draw upon the digital literacy metaphor, but make its shortcomings clear, and the latter two in particular extend the metaphor from a personal deficit model to a model that embraces technologies and social structures.

\section{Competency: what are the limits of instrumental literacy?}

Those who are functionally literate in the digital economy have solid instrumental literacy and make effective use of stabilised, well-domesticated digital applications. This group uses communication and transactional activities such as e-mail, online shopping, and online banking in a routine and effective way. 
In the initial stages of broadband use, these informants' Internet activities flow on seamlessly from previous experience and may be characterised as 'business as usual' (Wilken et al., 2011). For example, many participants had long experience paying bills and banking online, or searching Google for information, and used dial-up when this was the only technological platform available. Now of course, they use HSB and the NBN, but without augmenting these traditional activities that rely on instrumental literacy with those that might exercise semantic or social literacy:

'I still only use it for emails or finding bits of information, like recipes and that sort of stuff on the net, that's my main usage of it.'

'In dial-up times I was using the Internet for basic transactions ... it is certainly faster and more effective with higher [faster] broadband.'

'We are not hugely technologically minded people, so we tend to use it for the things we need to do rather than playing around with it for fun.'

'We use it just for a bit of gaming, standard emails and surfing, a bit of video and stuff like that. But it's pretty standard fare'

Despite increased speed and capacity, and despite an instrumental literacy strong enough to underpin access to services utilising semantic and social literacy, this group showed little inclination to do so. We suspect that this is not a consequence of a deficit, but is a consequence of a rational decision to deploy the NBN in some ways, but not in others, at this point in their lives, and at this point in the NBN's life. But this is not to say that there was no change at all in their digital habits, and their corpus of applications and practices has incrementally aggregated to embrace a somewhat expanded range of what remain prosaic and instrumental applications. Thus we saw our informants making online purchases from eBay as well as Wotif, transferring money across online accounts as well as paying bills online, or communicating through Skype as well as email.

This expansion may be in part a consequence of the increased 'legibility' of the digital corpus under the NBN. Participants did not have the same problems with speed or disconnections that were suffered under dial-up and ADSL, and the digital corpus was thus more tractable and convenient to domesticate. The expectation of the NBN was that it would do what the Internet had always done, but faster and more reliably. The NBN was an 
instrument that was useful in a limited range of applications, and was not imagined as a technology that would open up new worlds:

'I don't think it'll change what we do. I only use it for research and stuff. In the past if I wanted to buy something like a new chainsaw I'd trek around the retailers, now I just sit on the chair and get them up on the screen and see who's doing the best deal.

'It's not that much different. The faster speeds might make a difference for business, save a lot of time. But for us it's not really an issue. It's not the sort of thing we sit on for half a day.'

'I probably have enough computing power on my computer to design the space shuttle and I use it $90 \%$ of the time as a word processing machine. And the speed of the internet I could probably do something extraordinary but I won't use it...'

'I can see it going in the direction that they can go onto the TV and do maths homework or download educational stuff, or you can do your Woolworths shopping and things like that through the TV through the Internet, and being able to watch all these streamed shows with no issues about drop-outs and freezing and speed problems.'

\section{Legibility: is the NBN illegible?}

The exercise of literacy implies legible literature, and in these cases we find that effective use of the NBN is challenged, not because the user is digitally illiterate, but because the digital literature is illegible and incoherent. Where this is the case, the incoherence of the NBN at an instrumental level prevents engagement on a semantic or social level.

Of course, the NBN is not alone in this. People commonly feel that technology is difficult to understand, or unreasonable and irrational in its demands, despite the efforts of interface designers and software engineers to create 'user-friendly' products. Compounding the problem of technologies that are illegible in themselves, is the problem of the rapid versioning of digital technologies within lines, and the rapid redundancy of those lines. Unlike text literacy, it is as though one is no sooner familiar with a given text, than one finds it presented in a different dialect, or even a different language. 
Our informants reflected the difficulty in maintaining the legibility and coherence of digital technologies through the accumulation of devices in their homes, and the difficulties encountered with problems of interoperability of devices, and their continued functioning over time.

In many cases we saw homes littered with screens and devices of different ages, functionality, working order, and operating platforms. Often these new technologies appeared to be adopted by default, and the accumulation of new devices and services was accepted as part and parcel of contemporary living. Short life cycles, quick turn-over and high redundancy rates would appear to be a digital industry standard.

But the potential price paid for this is a collection of digital artefacts that are instrumentally illegible and semantically and socially incoherent. An issue that arose repeatedly, and mediated HSB expectations, involved difficulties in reading the demands of technologies and making them work; despite the promise of affordances providing for effective use, we discovered that, in practice, our informants found it difficult to get everything smoothly integrated and working as they would like:

'The wireless router is down there [downstairs office], and if I sit on this end of the lounge it's okay, but if I sit on that end it drops out ... it's really frustrating...it drops out and you lose everything.'

'Today is the first time the external hard drive has been connected to the laptop, I wanted to see if it could find it [music], so that I could then play the music that is on there through the Playstation through the stereo, but nothing works.'

'Certainly the NBN is faster, there is no doubt about that ... but the Wi-Fi diminishes the speed of the service undeniably. If I bring my notebook down here and put it right next to the modem it's a lot faster than if I work up in my office...it has that phrase "up-to”.'

Moreover, the illegibility of the NBN gives rise in some instances to an outright misreading of fundamentals - such as the difference between infrastructure and service provision:

'A lot of people just didn't understand the NBN. For instance my neighbour over the road, she actually got the letter... and she thought what she was paying for was the actual service - we explained to her it was like having your phone line laid but you 
didn't have to have a phone on at the moment. And when she realised that she said, "oh I should have filled it in".'

In the context of illegibility it is interesting to note that NBN Co has announced it is launching an education campaign for 2012 to explain what the rollout will mean for Australians, how to connect to the network, and why it is important that the nation upgrades its telecommunications infrastructure.

\section{Context: what are the structural conditions for literacy?}

The NBN is taking a place in an environment already populated with other technologies, with laws and regulations, with private and public institutions, and with all the other structural features that make up the social topography and technical ecosystem of a contemporary society. A powerful and large-scale actor though it is, the NBN has to find a way to work with or against, around or within, the social structures that precede it in the communications sector. Consistent with commercial and social norms in Australia, users have been required to opt-in to the initial NBN rollout, not opt-out, despite the fact that it represents itself as universal infrastructure, and despite the fact that it passes within meters of $93 \%$ of Australian homes. ${ }^{6}$ NBN had little choice in the matter, and nor do they have any choice in another feature of the structural landscape - home ownership, tenancies and property rights. We found that many people did not sign-up to the NBN, not because of digital illiteracy, and not because HSB or the NBN were illegible. Instead, because they were tenants and under property laws had no right to authorise the NBN Co to install the HSB hardware, this added a further layer of complexity for understanding the NBN installation process that compounded issues for literacy:

'There was a bit of a problem with Midway Point, because I think it is about fifty percent of the houses here are rentals, and you needed signatures from the owners for the box to be put onto the building.'

'I haven't looked into getting the new HSB only because we are renting and I thought opening that can of worms with my landlord would be a little bit difficult.'

'We got a lot of leaflets, but I found a lot of them quite confusing as to what the processes were getting connected if you were just renting...I think there was a form 
you could fill out to pass onto your landlord to get that sorted but I missed that, and then I got confused because I started getting other things saying you could easily connect. But I was under the assumption that if you hadn't made that initial approach and organised to get the house connected you'd have to do that first before signing up to something, so I found the process a little confusing.'

Whilst the opt-in model of early release sites is set to be overhauled by NBN Co, difficulties in understanding, communicating and establishing installation and service provision in the context of the structural landscape remains an issue. NBN uptake is not simply a matter of people waiting for their ISP contracts to expire, or their Landlords to sign connection agreements, but more significantly for the NBN, it also relates to confusing installation logistics, uncertainty in a complex and still fluid retail and technological environment, and a failure to communicate a clear 'value proposition' to the market:

'The NBN has umbrella companies, there's one part of NBN which hooks from the telegraph pole to that, and then there are subcontractors who hook this internal box to that, and then there's a third company involved to bring it all together. So it's just a mishmash.'

'So far they have failed to deliver what they said they would deliver. I'm not sure if it's NBN or iPrimus, but what we signed up for was 25 Megabits per second and it's gone from 2 to 5 recently. So 5 is as good as they are providing.'

'We spend $\$ 79$ per month...the NBN box is connected but it is just sitting there and I'll wait and see, it just depends but at the moment we don't have a need for it. I've got 200 gigs at the moment and the speed's good...'

'The only reason I don't swing onto it is I'm used to what we've got and if it's not busted why fix it...'

'We're on ADSL2+ whatever that means. I'm not 100\%, no even $50 \%$ up to date with speeds, it suits us, and it doesn't seem slow...'

\section{Conclusion}


The home will be a crucial site for the success of the NBN and growth of the digital economy (Apperley et al., 2011). The installation of fibre-to-the-home is often likened to last century's provision of electricity, and in this analogy the digital infrastructure of the twentyfirst century is expected to connect and integrate homes to the digital economy through the provision of universal, equitable and affordable high-speed broadband (HSB).

Next-generation broadband will enable video streaming applications, high-quality videoconferencing, faster and larger file transfers, and a number of high-bandwidth applications to run simultaneously (DBCDE, 2011; Ezell et al., 2009); and in the transformative vision of the NBN, the home will become smart and connected. While the notion of the connected or smart digital home has been around in different guises for a number of decades (see, for example, Edwards et al., 2011; Spigel, 2001; Venkatesh, 2008), it has been concretised in the wake of the NBN as a platform connected to the economy. The digital home is imagined as an integral part of a network of digital living with seamless transitions between home, office, supermarket, school, mall, and hospital; yet, the successful integration of the digital home as a critical platform within the digital economy relies upon a complex of shifting and interdependent literacies.

We have suggested in this paper that digital literacy has instrumental, semantic and social elements, and that effective use of HSB through the NBN requires more than the instrumental literacy necessary to provide access. We have argued that, in an information economy, the disadvantage that accrues from digital illiteracy is akin to the disadvantage that accrues from textual illiteracy, and in this sense the metaphor is useful. However, in so much as digital literacy and illiteracy focuses entirely on the presence or absence of personal skills, it is a deficit model that fails to account for other salient factors. We continue to deploy the literacy metaphor, but extend it beyond a deficit model by incorporating our observations of illegible technologies and services, and the structural conditions that deny the foundation upon which literacy might be built.

\section{Future research}

The tentative findings of the research study reported here suggest the need for further research, and we are currently pursuing questions of digital literacy as part of an ongoing study on the impact of broadband consumption on household media ecologies in the NBN 
release site of Brunswick, Victoria. Of particular interest will be the costs and benefits to family households associated with bandwidth plans, with the provision of new applications and services directed at media consumption, and with participation in the digital economy. We will address these questions by conducting a longitudinal survey of residents, and interviewing a smaller sample group of families, to document changes in the household media and communicative ecology (e.g. Hearn and Foth 2007; Shepherd et al., 2007; Tacchi, 2006), as well as consumption patterns, motivations and costs as HSB is habituated in the home.

The conceptual model of a media and communications ecology - of which some of the present authors have been instrumental in developing in relation to household environments (Shepherd et al., 2007) - is useful for situating more limited models of diffusion in relation to the physical and digital environments in which technology use and mediation takes place. The ecological metaphor shifts the focus away from studies focusing on discrete individual, devices or applications, to encompass systems of media and communications interaction. Thus, not only does the successful integration of the NBN with the digital home rely upon take-up of technical infrastructures and networks, but to fully realise ambitions for digital inclusion and the digital economy it also depends upon the aggregation and interaction of the household media ecology, including its technologies as well as its literacies and uses.

The implications of these findings for broadband policy, delivery and research on HSB, are that fibre networks cannot be reduced to a network of technologies, but are instead part of a much more heterogeneous network of actors that include legislation, landlords, ISP contracts, modems, literacies, and so on. Fibre-to-the-home in itself does not comprise the HSB network. Thus implementation policies and processes must go beyond physical installation to address the cultural contexts and participants that mediate delivery and adoption of broadband projects. This is recognised in the roll-out out by NBN Co of an education campaign - in parallel to their infrastructure roll-out - to explain how to connect to the network, why it is important to upgrade telecommunications infrastructure, and what HSB will mean for Australians. 


\section{References}

Apperley, T., Nansen, B., Arnold, M., and Wilken, R., 2011, 'Broadband in the Burbs: NBN Infrastructure, Spectrum Politics and the Digital Home', M/C Journal, XIV, 4, http://journal.media-culture.org.au/index.php/mcjournal/article/viewArticle/400.

Arnold, M., 2004, 'The Connected Homes Project: Probing the Effects and Affects of Domesticated ICTs', Proceedings of the Eighth Biennial Participatory Design Conference, 27-31 July, Toronto, Canada.

Arnold, M., Gibbs, M., and Shepherd, C., 2006a, 'Domestic ICTs, Desire and Fetish', Fibreculture Journal, 9, http://journal.fibreculture.org/issue9/issue9_arnold_gibbs_shepherd.html.

Arnold, M., Shepherd, C., Gibbs, M., and Mecoles, K., 2006a, 'Domestic Information and Communication Technologies and Subject-Object Relations: Gender, Identity and Family Life', Journal of Family Studies, 12, pp. 95-112.

Australian Communications and Media Authority (ACMA), 2007, Media Literacy Concepts, Research and Regulatory Issues, ACMA, Melbourne.

Australian Communications and Media Authority (ACMA), 2008, Developments in Internet Filtering Technologies and Other Measures for Promoting Online Safety: Second Annual Report to the Minister for Broadband, Communications and the Digital Economy, ACMA, Melbourne.

Australian Communications and Media Authority (ACMA), 2009, Digital Media Literacy in Australia Key Indicators and Research Sources, ACMA, Melbourne.

Conroy, S. (Minister for Broadband, Communications and the Digital Economy), 2009, New National Broadband Network, joint media release, Parliament House, Canberra, http://www.minister.dbcde.gov.au/media/media_releases/2009/022.

Department of Broadband, Communications and the Digital Economy (DBCDE), 2011, Submission to the House Standing Committee on Infrastructure and Communications: The Role and Potential of the National Broadband Network. Canberra: DBCDE.

Edwards, K. W., Grinter, R. E., Mahajan, R., and Wetherall, D., 2011, 'Advancing the State of Home Networking', Communications of the ACM, LIV, 6, pp. 62-71.

European Commission, 2009, Audiovisual and Media Policies, http://ec.europa.eu/comm/avpolicy/media_literacy/index_en.htm.

Ezell, S., Atkinson, R., Castro, D., and Ou, G., 2009, 'The Need for Speed: The Importance of Next-Generation Broadband Networks', The Information Technology \& Innovation Foundation, Washington, DC, http://www.itif.org/files/2009-needforspeed.pdf

Foth, M., Forlano, L., Scatchell, C., and Gibbs, M., (eds) 2011, From Social Butterfly to Engaged Citizen: Urban Informatics, Social Media, Ubiquitous Computing, and Mobile Technology to Support Citizen Engagement, MIT Press, Cambridge, MA. 
Gaver, W., Boucher, A., Pennington, S., and Walker, B., 2004, 'Cultural Probes and the Value of Uncertainty', Interactions, XI, 1, pp. 53-56.

Gaver, W., Dunne, A., and Pacenti, E., 1999, 'Design: Cultural Probes', Interactions, VI, 1, pp. 21-29.

Given, J., 2010, “"The Most Connected Place on the Planet”, Communication, Politics \& Culture, XLIII, 1, pp. 120-142.

Hearn, G., and Foth, M., 2007, 'Communicative Ecologies: Editorial Preface', Electronic Journal of Communication 17(1-2).

Gurstein, M., 2003, 'Effective Use: A Community Informatics Strategy Beyond the Digital Divide', First Monday, XIII, 12, http://firstmonday.org/htbin/cgiwrap/bin/ojs/index.php/fm/article/view/1107/1027.

Gurstein, M., 2004, 'Effective Use and the Community Informatics Sector: Some Thoughts on Canada's Approach to Community Technology/Community Access', in M. Moll and L. Shade, (eds), Seeking Convergence in Policy and Practice: Communications in the Public Interest Vol. 2, Canadian Centre for Policy Alternatives, Ottawa, pp. 223-243.

Hobbs, R., 2008, 'Debates and Challenges Facing New Literacies in the $21^{\text {st }}$ Century', in K. Drotner and S. Livingstone (eds), International Handbook of Children, Media and Culture, Sage, London, pp. 431-447.

Jenkins, H., 2006, Convergence Culture: Where Old and New Media Collide, New York University Press, New York.

Jenkins, H. (with Purushotma, R., Clinton, K., Weigel, M. and Robison, A.J.), 2009, Confronting the Challenges of Participatory Culture: Media Education for the 21st Century, MacArthur Foundation, MIT Press, Cambridge, MA.

Livingstone, S., 2009, Children and the Internet: Great Expectations, Challenging Realities, Polity, Cambridge.

Luke, C., 2000, 'Cyberschooling and Technological Change: Multiliteracies for New Times', in M. Kalantzis and B. Cope (eds), Multiliteracies: Literacy, Learning and the Design of Social Futures, Cambridge University Press, Cambridge, pp. 69-91.

Luke, C., 2001, 'New Times, New Media: Where to Media Education?' Media International Australia, 101, pp. 87-100.

Maher, A., 2008, Digital Inclusion Initiative, Melbourne: Infoxchange

Rogers, E. M., 1983, Diffusion of Innovations, third edition, Free Press, New York.

OfCom, 2009, Report of the Digital Britain Media Literacy Working Group, UK, http://stakeholders.ofcom.org.uk/binaries/research/media-literacy/digitalbritain.pdf.

Satchell, C., and Dourish, P., 2009, Beyond the user: use and non-use in HCI. In Proceedings of the 21st Annual Conference of the Australian Computer-Human Interaction Special Interest Group (OZCHI '09) New York: ACM, pp. 9-16. 
Selwyn, N., 2003, 'Apart from Technology: Understanding People's Non-Use of Information and Communication Technologies in Everyday Life', Technology in Society, 25, pp. 99-116.

Shepherd, C., Arnold, M., Bellamy, C., and Gibbs, M., 2007, 'The Material Ecologies of Domestic ICTs', The Electronic Journal of Communication, XVII, 1-2, http://www.cios.org/www/ejc/v17n12.htm.

Spigel, L., 2001, 'Media Homes: Then and Now', International Journal of Cultural Studies, IV, 4, pp. 385-411.

Spring, J., and Wiatr, R., 2006, 'The TasCOLT Project', Telecommunication Journal of Australia, LVI, 3/4, pp. 37-48.

Tacchi, J., 2006, 'Studying Communicative Ecologies: An Ethnographic Approach to Information and Communication Technologies (ICTs)', In Proceedings $56^{\text {th }}$ Annual Conference of the International Communication Association, Dresden, Germany.

Tasmanian Electronic Commerce Centre Pty Ltd (TECC), 2008, 'Report on the Rollout of the tasCOLT Fibre to the Premises Commercial Trial', Tasmanian Electronic Commerce Centre Pty Ltd, Hobart, http://tecc.additionalview.com/wp-content/uploads/2009/07/TECCtasCOLT-Review-Report-20081117.pdf

Venkatesh, A., 2008, 'Digital Home Technologies and Transformation of Households', Information Systems Frontiers, 10, pp. 391-395.

Verbeek, P., 2005, What Things Do: Philosophical Reflections on Technology, Agency, and Design. Pennsylvania State University Press.

Warschauer, M., 2003, Technology and Social Inclusion: Rethinking the Digital Divide. Cambridge: MIT Press.

Wilken, R., Nansen B, and Arnold M., 2011, 'Broadband in the Home Pilot Study: Suburban Hobart., Telecommunications Journal of Australia 61.1: 5.1-5.16.

\section{Notes}

1 Funded by The Institute for the Broadband Enabled Society (IBES), The University of Melbourne, and with in-kind support provided by Hybrid TV.

2 Funded by The Australian Communications Consumer Action Network (ACCAN).

3 Four households were made up of families with a mother, father and either two or three children aged between 8 and 16: two households had two parents and a single teenage child; two households were couples without children; one house was a single father and teenage son; and one house was a couple with a young child (Wilken at al., 2011).

4 Each of the participants in the Hybrid SmartStreet project were given a TiVo media device that would permit access to high definition TV, as well as existing broadband services via Hybrid TV's Video-onDemand (VoD) entertainment service, CASPA (TECC, 2009), as well as free Internet service for 12 months. While this trial was meant to simulate the NBN in terms of Internet speed and capacity over the 
period of the trial, our participants have reported broadband speeds up to $4 \mathrm{Mbps}$ rather than the projected 100 Mbps. The broadband speed provided is, nonetheless, a faster service than our participants previously had - often dial-up - and so can speak to trends in domestic broadband use following increased bandwidth provision and domestication.

5 Composition of households was mixed: 15 owned their home, 5 rented; 9 households were families with children, 6 were couples, 4 were shared houses and 1 was a single occupant; 12 households were connected to the NBN, 6 were connected to other broadband services and 2 had wireless connectivity.

6 This 'opt-in' policy differs in Tasmania where state legislation passed in 2010 shifted to an opt-out policy. 